\title{
Left ventricular diverticula in a 2-year-old child with a life-threatening ventricular arrhythmic manifestation - Treatment strategy and review of the literature
}

\author{
Wojciech Szychta $^{1 *}$, Grażyna Brzezińska-Rajszys ${ }^{1}$, Łukasz Szumowski ${ }^{2}$, Monika Kowalczyk-Domagała ${ }^{1}$, Maria Miszczak-Knecht ${ }^{1}$ and \\ Katarzyna Bieganowska ${ }^{1}$ \\ ${ }^{1}$ The Children's Memorial Health Institute, Poland \\ ${ }^{2}$ Institute of Cardiology, Poland
}

\begin{abstract}
\section{Introduction}

Congenital outpouching of heart chambers is a rarely found pathology that includes congenital ventricular diverticulum (CVD) and aneurysm (CVA) [1,2]. The pathologies are usually found during transthoracic echocardiography (TTE). The most reproducible parameter for diagnosis of CVD in TTE is contractility of the pathological chamber. The frequency of occurrence is $0.013 \%-0.76 \%$ of patients undergoing echocardiographic examination and $0.4 \%$ of autopsies after cardiac death [2-4]. Their true incidence is unknown. This pathology is very unusual even in the pediatric population [1]. Only a few long-term observational studies and some case reports have been published considering this pathology [3-7]. CVD, in contrast to CVA, is usually a benign malformation [2]. We present a child with the left ventricle diverticulum (LVD) who had a life-threating ventricular arrhythmia due to the congenital pathology.
\end{abstract}

Left ventricular diverticulum (LVD) is a rarely found and tricky pathology. The diagnosis in echocardiography lies in synchronous contractility. We present a child with LVD and coexisting life-threating ventricular arrhythmia. The patient was qualified to ablation of arrhythmia and conservative treatment of the diverticulum itself. The girl remains in a good clinical condition after 10 years of follow-up. However, a recent Holter ECG examination revealed benign ventricular arrhythmia.

\section{Case report}

We present a 2 year 8 months old girl, $13.4 \mathrm{~kg}$ of weight, who was referred for a second opinion with the diagnosis of heart tumor and a complex ventricular arrhythmia. She had a history of two episodes of loss of consciousness. The second one was witnessed by a paramedic who noted an irregular heart rhythm at the time of the event. The electrocardiogram (ECG) showed sinus rhythm, normal axis, multiple ventricular extrasystoles (VEs) with a morphology of the right bundle branch block (RBBB) and a left axis deviation as well as 1 triplet of VE $100 /$ min (Figure 1). In the Holter ECG examination, which was performed on two antiarrhythmic drugs (amiodarone $70 \mathrm{mg}$, mexiletine $180 \mathrm{mg} / 24 \mathrm{~h}$ ), we noted $38691 \mathrm{VEs}$ (32\% of all QRS), 1724 non-sustained ventricular tachycardia (nsVT) (up to 10 subsequent beats and maximal heart rate 205/min) (Figure 2). Moreover, constant ECG monitoring revealed sustained ventricular tachycardia with maximal heart rhythm $280 / \mathrm{min}$ followed by a spontaneous return of sinus rhythm (Figure 3). TTE revealed a large contractile pouch with a broad connection to the ventricle close to the mitral valve (MV) ring.
The contractility of the outpouching area was asynchronous (Figures 4 and 5). Cardiac catheterization procedure confirmed the diagnosis and revealed the LVD surrounding the MV (Figure 6).

Our cardiology team consulted the patient and qualified to radiofrequency ablation because of the life-threatening cardiac arrhythmia. Fluoroscopy integrated 3D mapping of the left ventricle showed that the origin of the ventricular arrhythmia was at the entrance to the LVD. Several radiofrequency applications were applied there. Post ablation control electrophysiological study with aggressive stimulation program did not induce any VEs. After the procedure, some Holter ECG examinations showed that the arrhythmia was still present, but less significant. The antiarrhythmic therapy was intensified (amiodarone $100 \mathrm{mg}$, mexiletine $150 \mathrm{mg} / 24 \mathrm{~h}$ ) by adding propranolol ( $45 \mathrm{mg} / 24 \mathrm{~h}$ ) which led to a further reduction of the arrhythmia.

During the follow-up period, the patient was observed in the outpatient clinic. The girl was in a good clinical condition with no symptoms. She led an active lifestyle and participated in sports activities. In subsequent Holter ECG studies, a reduction of arrhythmia was observed, and pharmacotherapy was slowly discontinued. During the last visit, 10 years after the ablation procedure, ventricular arrhythmia again appeared, effort suppressed arrhythmia. The patient the patient remains under our outpatient clinic without medication.

${ }^{*}$ Correspondence to: Wojciech Szychta, The Children's Memorial Health Institute, Dzieci Polskich 20, Warsaw, Poland, Tel: +48 2281573 70, Fax: +48 2281573 65; E-mail: wszychta@wp.pl

Key words: left ventricular aneurysm, congenital heart disease, ventricular tachycardia, cardiac imaging, pediatric echocardiography, percutaneous intervention

Received: August 30, 2019; Accepted: September 16, 2019; Published: September 20, 2019 
Szychta W (2019) Left ventricular diverticula in a 2-year-old child with a life-threatening ventricular arrhythmic manifestation - Treatment strategy and review of the literature

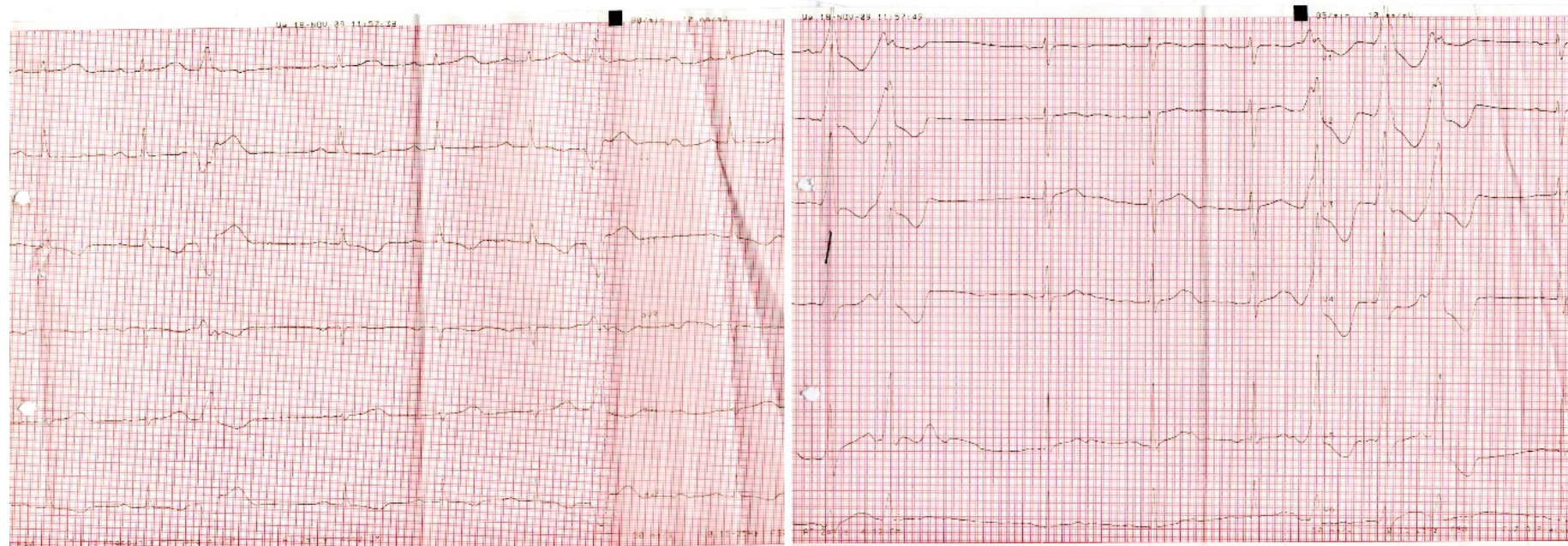

Figure 1. ECG of the patient on the day of admission to the hospital

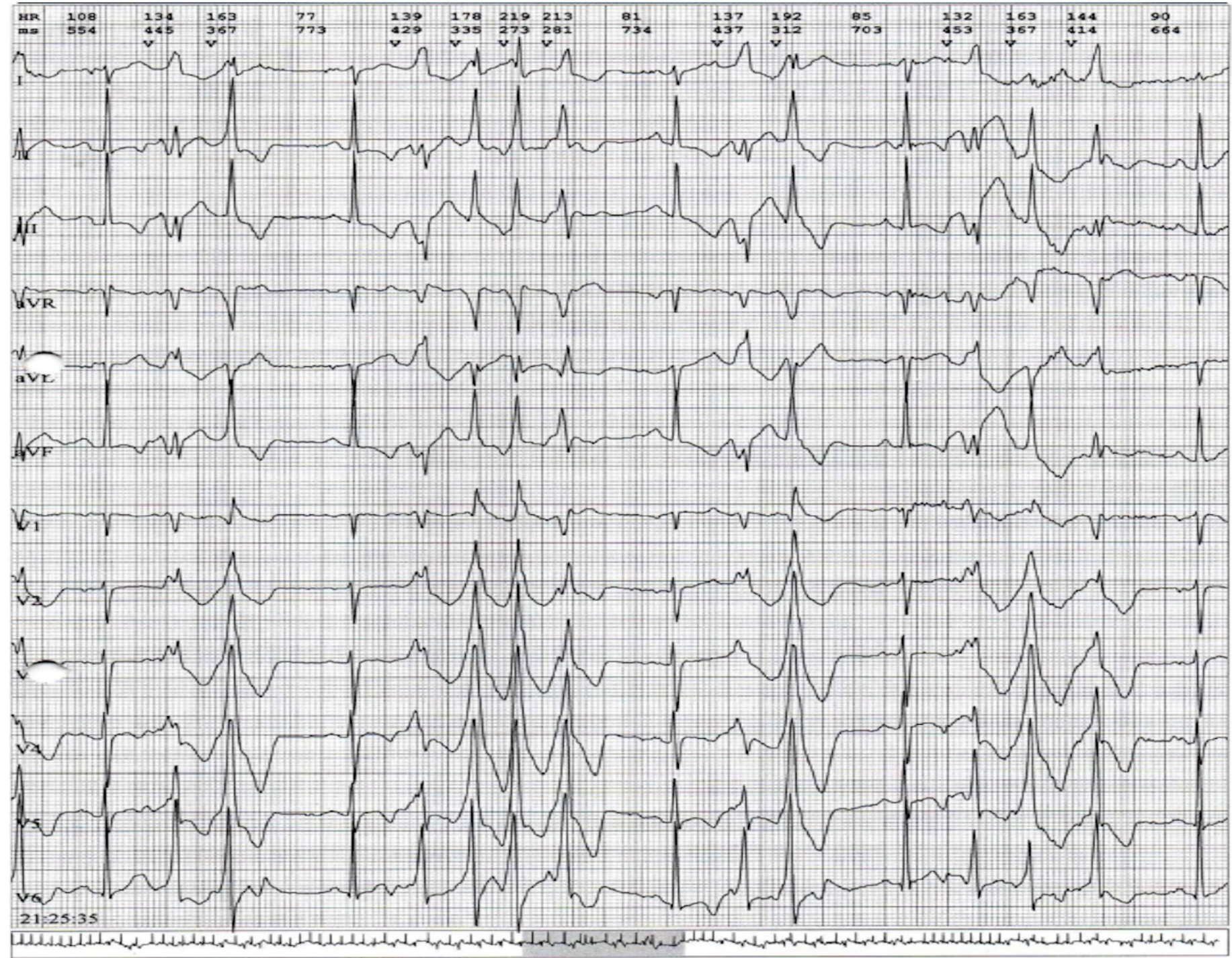

Figure 2. Complex ventricular arrhythmia in Holter ECG of the patient 
Szychta W (2019) Left ventricular diverticula in a 2-year-old child with a life-threatening ventricular arrhythmic manifestation - Treatment strategy and review of the literature

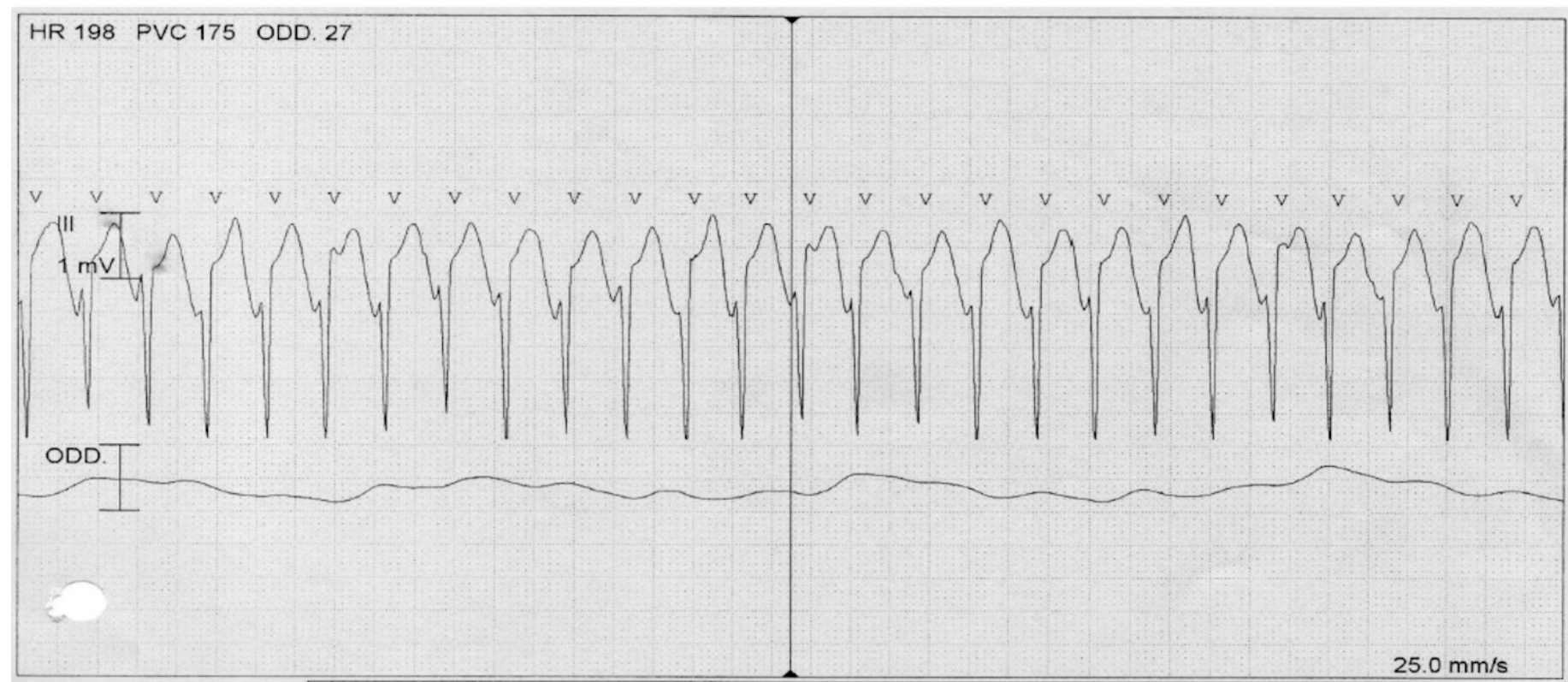

Figure 3. Constant monitoring: sustained ventricular tachycardia

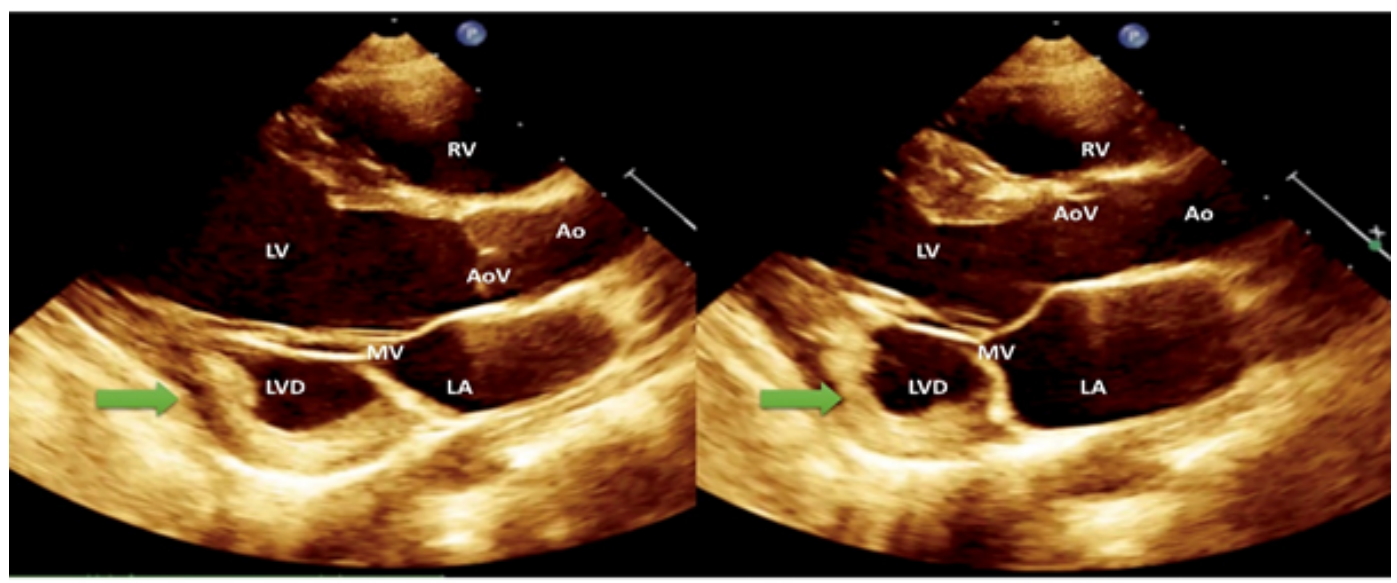

Diastole

Systole

Figure 4. Large contractile pouch - LVD with a broad connection to the LV. Asynchronous contraction of the LVD. LAX TTE.

Ao - aorta; AoV - aortic valve; LA - left atrium; LAX - long axis; LV - left ventricle; LVD - left ventricle diverticulum; MV - mitral valve; RV - right ventricle; TTE - transthoracic echocardiogram

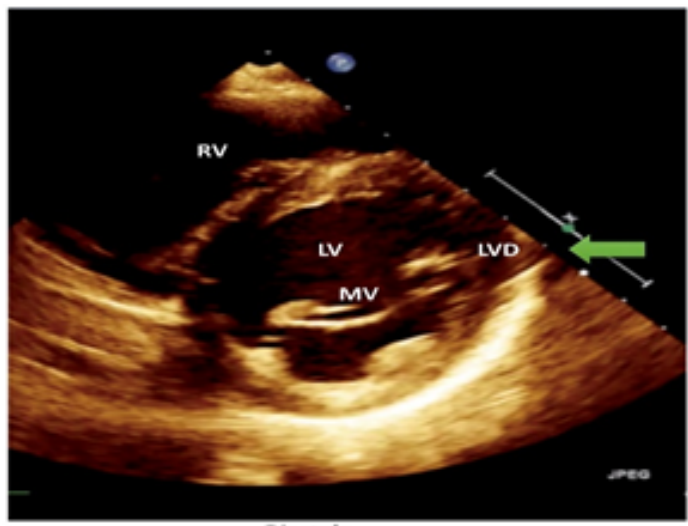

Diastole

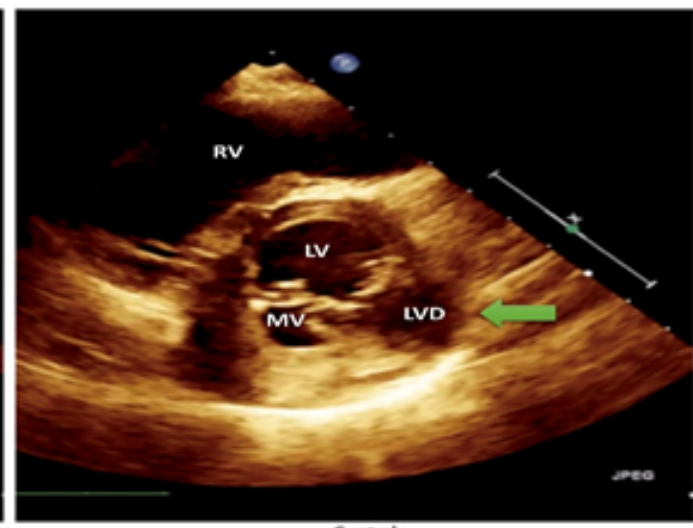

Systole

Figure 5. Large contractile pouch - LVD surrounding the MV. Asynchronous contraction of the LVD. SAX TTE. LV - left ventricle; LVD - left ventricle diverticulum; MV - mitral valve; $\mathrm{RV}$ - right ventricle; SAX - short axis; TTE - transthoracic echocardiogram 


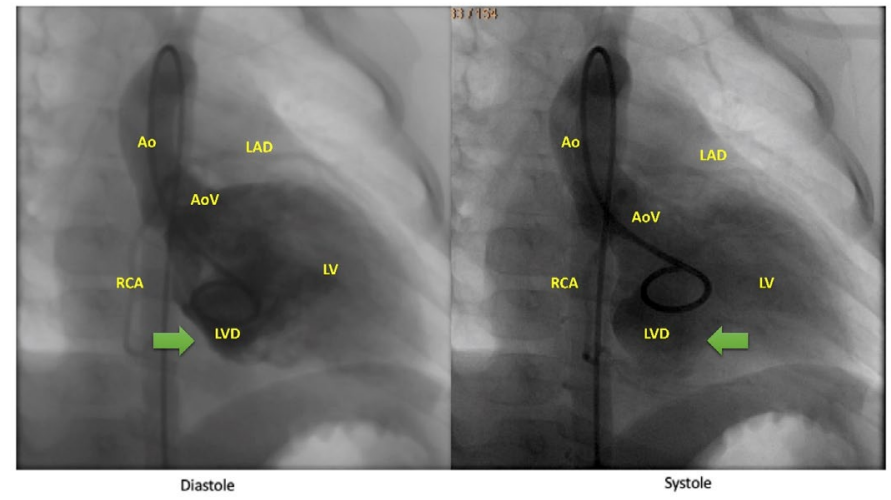

Figure 6. Cardiac catheterization procedure - LVD surrounding the MV. Asynchronous contraction of the LVD. Ao - aorta; AoV - aortic valve; LAD - left anterior descending artery; LV - left ventricle; LVD - left ventricle diverticulum; RCA - right coronary artery

\section{Comment}

We present a rare case of a very young child diagnosed due to life-threating ventricular arrhythmia with a diagnosed LVD who had fully recovered after ablation, which to the best of our belief, has never been described before. There are a few articles published previously considering LVD with sustained ventricular arrhythmia, but all of them are considering adult patients [2-7]. Most patients with the diagnosis of LVD have no symptoms. However, such complications as embolism, arrhythmias, cardiac failure, rupture and compression of a coronary artery can be found in the clinical setting [1-3]. Ventricular arrhythmia coexisting with LVD/A is a rare finding and can be found in up to $13 \%$ of adult patients with this congenital pathology [2]. In the pediatric population about $20 \%$ of patients with LVD/A has ventricular arrhythmia [1-6]. Whereas $90 \%$ of those cases are documented as ventricular tachycardia (VT) and sustained VT [2-5]. A ventricular arrhythmia may cause palpitations, syncope or even sudden cardiac death. Our patient's symptoms.

Echocardiography is the most commonly used diagnostic method in the diagnosis of CVD/A [1,2]. It is a challenging pathology as nonapical CVDs are often misdiagnosed. However, improvement in the availability of fetal echocardiography is needed as up to $62.5 \%$ of cases can be diagnosed on prenatal examination [1-6]. Marijon et al. [1] noted 22 pediatric patients with CVD/A over 31 years of follow-up. 16 of them had CVD and 6 CVA diagnosed. CVD is usually diagnosed in the ventricular apex (8 cases); however, it can be found in other ventricular segments. Non-apical CVDs are usually large contractile outpouching areas with a broad connection to the ventricle and are not associated with midline thoracoabdominal defects or other intracardiac abnormalities. On the other hand, apical CVDs are always associated with cardiac rotation disorders (mesocardiac or dextrocardia) and/or intracardiac abnormalities [1]. This pathology can be challenging to differentiate, and cardiac magnetic resonance with delayed contrast enhancement can be needed to verify the occurrence of fibrosis which is typical for CVA [3].

Management of such patients is challenging. Having the patient in severe clinical condition due to ventricular arrhythmia we decided to perform an ablation procedure guided by electroanatomic imaging (CARTO) as the first line treatment. It was a high-risk procedure, due to severe arrhythmia, but also due to the very young age and a small weight of the patient. Therefore, it was performed in a hybrid operating theatre having a cardiac surgeon and a perfusionist ready to perform on-pump surgery during the whole procedure. The pathology itself is usually not an indication for surgical treatment [1].

In most cases, observation in outpatient clinics can be justified by a quite good prognosis of patients with CVD. More than $80 \%$ of them are alive at 10 years of follow-up. On the other hand, the prognosis for CVA patients is poor, with $30 \%$ survival after 4 years of observation [1]. Such an observation is in contrast with our patient whose prognosis was poor before ablation. However, after 10 years of follow-up, the girl remains in a good clinical condition. During the last visit in our out-patient clinic the girl did not report any disturbing symptoms, was in good condition, but we found benign ventricular arrhythmia in Holter's ECG examination which was surprise for us. So further longterm observation is necessary.

\section{Conclusion}

A left ventricular diverticulum can coexist with a life-threatening ventricular arrhythmia and ablation procedure may be necessary even in small patients. Patients with ventricular arrhythmia and LVD require long term follow-up because recurrence of arrhythmia is possible.

\section{References}

1. Marijon E, Ou P, Fermont L, Concordet S, Le Bidois J, et al. (2006) Diagnosis and outcome in congenital ventricular diverticulum and aneurysm. $J$ Thorac Cardiovasc Surg 131: 433-437. [Crossref]

2. Haegeli LM, Ercin E, Wolber T, Brunckhorst C, Tanner FX, et al. (2011) Arrhythmic manifestations in patients with congenital left ventricular aneurysms and diverticula Am J Cardiol 108: 1826-1830. [Crossref]

3. Kumar PV, Moorthy A (2016) An unfortunate case of subaortic left ventricular diverticulum. Indian Heart J 68: S110-S113. [Crossref]

4. Bhardwaj B, Kumar SA, Webel R, Gautam S, Chockalingam A (2019) Multimodality detection of multiple left ventricular diverticula: A case report and brief review of the literature. Echocardiography 36: 184-188. [Crossref]

5. Sierra M, Huynh H, Machado C (2009) Congenital ventricular diverticulum presenting as sustained monomorphic ventricular tachycardia. Int $J$ cardiology 133: e70-72. [Crossref]

6. Peters C, Wacker-Gussmann A, Strasburger JF, Cuneo BF, Gotteiner N et al. (2015) Electrophysiologic features of fetal ventricular aneurysms and diverticula. Prenat Diagn 35: 129-136. [Crossref]

7. Martini B, Trevisi N, Martini N, Zhang L (2015) Right ventricular outflow tract tachycardia with structural abnormalities of the right ventricle and left ventricular diverticulum. Case Rep Cardiol 2015: 708687. [Crossref]

Copyright: $\odot 2019$ Szychta W. This is an open-access article distributed under the terms of the Creative Commons Attribution License, which permits unrestricted use, distribution, and reproduction in any medium, provided the original author and source are credited. 